\title{
Statistical process control readiness in the food industry: development of a self- assessment tool
}

\begin{abstract}
Background: The increasing pressure from the customers, governmental regulations and fierce market competition forced food companies to pursue powerful quality improvement technique. Although Statistical Process Control is widely known for its effectiveness in process control, many food companies faced difficulties to adopt such technique, where being in the state of not ready has always been the reason. There has been a debate about the importance of deciding the state of readiness of a company to initiate their CI techniques such as SPC towards the successful implementation and sustainability of such technique.Scope and approach: This paper emphasises the importance of SPC readiness towards its implementation in the food industry and determines its factors. The SPC readiness factors were identified based on the current literature review and complemented with a three-round Delphi study involving the SPC experts (academics, industry and consultants). Key findings and conclusion: The SPC readiness factors identified are top management support, sense of urgency, measurement system, employees involvement and organisational culture readiness. The developed conceptual self-assessment readiness tool enables food practitioners to identify the current state of organisational readiness and facilitate the companies to plan strategic changes and preparation activities for the adoption of SPC in their businesses.
\end{abstract}

Keyword: Food industry; Organisational readiness; Statistical process control; Continuous improvement 